\title{
Bioprosthetic Valve Thrombosis
}

\author{
Sarina Sachdev ${ }^{\mathrm{a}}$, Nikky Bardia ${ }^{\mathrm{a}}$, Landai Nguyen ${ }^{\mathrm{a}}$, \\ Bassam Omara, b
}

\begin{abstract}
Degenerative valve disease is on the rise with greater than 100,000 valve operations performed in the US alone per year. The majority of those procedures employ tissue bioprostheses to avoid the attendant risk of anticoagulation, especially in the elderly. Though traditionally this approach has been considered a superior option to avoid anticoagulation, more recent analyses have demonstrated a significant incidence of previously unrecognized thrombosis associated with bioprosthetic valves, especially with the more recent advent of the transcatheter aortic valve replacement implantations. Bioprosthetic valve thrombosis is a major cause of either acute or indolent bioprosthetic valve degeneration, and often has an elusive presentation causing delayed recognition and treatment. The literature has extensively addressed the risks and benefits of anticoagulation following bioprosthetic valve replacement to prevent bioprosthetic valve thrombosis (BPVT), without conclusive evidence-based recommendations. The duration of anticoagulation following an episode of BPVT is unclear, and lifelong anticoagulation has been suggested. The increasing use of transcatheter aortic valve replacement as an alternative to surgical aortic valve replacement in various risk groups has introduced new challenges with regards to valve thrombosis, which have been poorly studied with regards to optimal treatment and prevention. The increasing use of valve-in-valve procedures is expected to bring on further uncharted challenges.
\end{abstract}

Keywords: Bioprosthetic valve; Thrombosis; Valvular heart disease

\section{Introduction}

With the aging population in the US and elsewhere, degenerative valve disease is on the rise with greater than 100,000 valve operations performed in the US alone per year. The majority of those procedures nowadays (80-90\%) employ tissue bioprostheses to avoid the attendant risk of anticoagulation, especially

Manuscript submitted October 7, 2018, accepted October 25, 2018

aUniversity of South Alabama, Mobile, AL 36617, USA

${ }^{\mathrm{b}}$ Corresponding Author: Bassam Omar, Division of Cardiology, University of South Alabama, 2451 USA Medical Center Dr., Mobile, AL 36617, USA. Email: bomar@health.southalabama.edu

doi: https://doi.org/10.14740/cr789 in the elderly [1]. Though traditionally this approach has been considered a superior option to avoid anticoagulation, more recent analyses have demonstrated a significant incidence of previously unrecognized thrombosis associated with bioprosthetic valves [2], especially with the more recent advent of the transcatheter aortic valve replacement (TAVR) implantations [3].

\section{Epidemiology}

Valvular heart disease is fairly common, with prevalence in excess of $10 \%$ in patients older than 75 years of age [4]. With a rising rate of valve operations in the US and worldwide, various complications have been increasingly recognized, especially valve thrombosis [5], which has been reported to be on the rise even with the increasing use of the less thrombogenic biologic valves $[6,7]$.

Brown et al [8] analyzed 4,568 patients who received biologic valves for aortic valve replacement over 15 years and reported a significant incidence of early thrombosis of porcine aortic bioprostheses requiring reoperation: $1.26 \%$ for the Biocor valve, $0.37 \%$ for the Mosaic valve, and $0.84 \%$ for the Hancock valve. They found no cases of thrombosis for the pericardial valve $(5,923$ patient-years) or the stentless valve (172 patient-years).

Makkar et al [9] reported reduced leaflet motion on computed tomography (CT) in 22 of 55 patients $(40 \%)$ who received TAVR in the PORTICO IDE clinical trial and in 17 of 132 patients $(13 \%)$ in two TAVR registries (RESOLVE and SAVORY). Reduced leaflet motion was detected among patients with multiple bioprosthesis types, including transcatheter and surgical bioprostheses. Anticoagulation with warfarin, in contrast to dual antiplatelet therapy, was associated with a decreased incidence of reduced leaflet mobility $(0 \%$ and $55 \%$, respectively, $\mathrm{P}=0.01$ in the clinical trial; and $0 \%$ and $29 \%$, respectively, $\mathrm{P}=0.04$ in the pooled registries).

Chakravarty et al [10] further analyzed the subclinical leaflet thrombosis in bioprosthetic aortic valves, which was found to be more commonly in transcatheter than in surgical valves and associated with increased transient ischemic attack (TIA) and stroke risk. Anticoagulation, both with non-vitamin $\mathrm{K}$ antagonist (VKA) oral anticoagulants (NOACs), and warfarin, but not with dual antiplatelet therapy, was effective in preventing or treating subclinical leaflet thrombosis. They identified prevention and treatment of subclinical leaflet thrombosis as a potential opportunity to improve valve hemodynamics and clinical outcomes in TAVR. 
Table 1. Predictors of BPVT Reported in the Literature

\begin{tabular}{lll}
\hline Patient factors & Valve/surgical factors & Rheolytic factors \\
\hline High body mass index & Small valve size & Lack of anticoagulation, \\
Female gender & TAVR valve-in-valve (VIV) & Hypercoagulability \\
Atrial fibrillation & Suboptimal valve placement (TAVR) & Eosinophilia \\
Atrial dilatation & Non-resection of native valve & Oral contraceptives \\
low cardiac function & Aortic root morphology & Polycythemia \\
VA-ECMO & Calcified aortic annulus & HIT \\
Calcium supplements & & Fondaparinux \\
& & Kounis syndrome \\
\hline
\end{tabular}

BPVT: bioprosthetic valve thrombosis; VA-ECMO: venoarterial extracorporeal membrane oxygenation; VIV: valve-in-valve; TAVR: transcatheter aortic valve replacement.

Cordoba-Soriano et al [11] reviewed 11 publications reporting 16 patients with valve thrombosis at a median of 6 months following TAVR, 15 of whom received a balloon-expandable valve; all received dual antiplatelets following the procedure and continued to take either mono- or dual antiplatelets at the time of valve thrombosis diagnosis. Progressive dyspnea was the most common symptom and leaflet thickening and increase in transvalvular gradient (TVG) from 10 to 40 $\mathrm{mm} \mathrm{Hg}$ were the most common transthoracic echocardiogram (TTE) findings. Systemic embolism was not a post-TAVR feature of valve thrombosis.

Oliver et al [12] found mitral bioprosthetic valve thrombosis (BPVT) in 10 of 161 patients $(6.2 \%)$ with evidence of bioprosthesis dysfunction suggesting that BPVT has a higher incidence than was previously reported. Two patients underwent prosthetic valve replacements and the remainder received anticoagulation.

Egbe et al [13] identified BPVT in 46 out of 397 (11\%) bioprosthetic valves explanted at Mayo Clinic, which also suggested BPVT to be more frequent cause of bioprosthesis dysfunction. In contrast to the misconception that BPVT is a perioperative phenomenon, Egbe's study also revealed that $65 \%$ of all reoperations were due to BPVT, occurred more than 1 year after implantation, and up to $15 \%$ of these reoperations occurred more than 5 years after implantation.

\section{Natural History}

Many cases of BPVT, involving surgical and catheter-based implanted bioprosthetic valves, are subclinical and elusive, therefore evading early diagnosis and timely treatment, leading to early bioprosthetic valve failure [14].

Sondergaard et al [15] reported the analysis of four-dimensional volume-rendered CT (4D-CT) of patients treated with TAVR or surgical aortic valve replacement (SAVR) in the SAVORY registry, which revealed cases of hypo-attenuating leaflet thickening (HALT) with or without hypoattenuation affecting motion (HAM). Although the temporal pattern of these observations is unclear, overall subclinical leaflet thrombosis was common, progressing from normal leaflets to HALT or to the more pronounced HAM. These phenomena may appear or resolve at variable intervals after valve implantation, with some evidence of a protective effect of anticoagulants.

Doris and Dweck [16] suggested that bioprosthetic valve degeneration may be triggered by a mechanism involving subclinical leaflet thrombosis and secondary calcification of the leaflets, leading to subsequent degenerative changes and valve dysfunction.

\section{Pathophysiology}

Although no specific etiology stands out in the pathogenesis of BPVT, several valve characteristics and patient profiles, including comorbidities, have been associated with increased incidence of thrombus formation on bioprosthetic valves (Table 1).

Mack and Holmes [17] listed several potential predisposing factors for BPVT, including small valve size, high body mass index, lack of anticoagulation, and a TAVR valve in a previously placed surgical valve. They made three assertions: the first is that BPVT is more common than previously recognized, the second that $4 \mathrm{D}-\mathrm{CT}$ is the most sensitive imaging modality for detection, and the third is that anticoagulation can resolve the problem in some patients.

Imada et al [18] reported a 61-year-old male who required venoarterial extracorporeal membrane oxygenation (VA-ECMO) due to extremely low cardiac output following mitral valve replacement (MVR) with a tissue valve, and was found to have mitral stenosis due to bioprosthetic mitral valve thrombus on TTE prior to planned left ventricular assist device (LVAD) implantation. They offered additional potential explanations to BPVT including hypercoagulability, atrial fibrillation, atrial dilatation, low cardiac function, and lack of anticoagulation therapy. Madathil et al [19] reported a similar case in a 21-year-old male requiring VA-ECMO following double valve replacement who had fatal bioprosthetic mitral valve thrombosis 2 days postoperatively. Kagiyama et al [20] reported two other cases of a 75-year-old female and a 70-year-old male with acute BPVT of mitral valves in the setting of VAECMO, despite anticoagulation.

Kothari et al [21] reported a 31-year-old male with signifi- 
cant eosinophilia and dyspnea who was found to have an increased gradient across a mitral bioprosthetic valve implanted 7 years earlier, with thickened leaflets. Treatment with corticosteroids and anticoagulation resulted in resolution of symptoms and decrease in the gradient and leaflet thickening of the bioprosthetic valve.

Yesin et al [22] reported a 44-year-old female with a bioprosthetic mitral valve implanted 6 years earlier who presented with significant dyspnea and was found to have increased TVG and decreased valve area caused by a large thrombus, all of which resolved following treatment with thrombolytic therapy. Extensive workup revealed no genetic mutation or protein deficiency which may have caused a hypercoagulable state; therefore her BPVT was blamed on oral contraceptive drug therapy including ethinyl estradiol and desogestrel which she started taking 1 month before admission.

Poels et al [23] reported severe BPVT in a 69-year-old female presenting with progressive dyspnea and syncope 9 months following TAVR (Edwards-Sapien 23-mm prosthesis) who was successfully treated with bioprosthetic MVR. Laboratory studies revealed a heterozygote prothrombin gene mutation causing a hypercoagulable state with a strongly increased factor VIII; she was therefore treated with aspirin and warfarin.

Chamsi-Pasha et al [24] reported a 74-year-old female with dyspnea and fatigue 15 months following bioprosthetic MVR; transesophageal echocardiography (TEE) showed severe diffuse bioprosthetic mitral valve leaflet thickening with severely restricted motion, indicative of diffuse thrombosis, which was successfully treated with thrombolytics and anticoagulation. She was found to have isolated positive titers of anticardiolipin IgM antibodies, which remained positive on repeat testing 12 weeks afterward, and was therefore treated with long-term anticoagulation for antiphopholipid antibody syndrome.

Sidhu et al [25] reported a 72-year-old female with severe dyspnea and shock 7 days post bioprosthetic MVR who had a decline in platelet count with a positive heparin-induced thrombocytopenia (HIT) antibody assay; TTE and TEE both showed large immobile thrombi involving the left atrium and the mitral valve prosthesis. She underwent redo MVR with a mechanical prosthesis, but later died of multiorgan failure.

Tsiouris et al [26] reported a 75-year-old male with dyspnea and shock due to acute BPVT and obstruction of a mitral valve prosthesis implanted 2 months earlier; he recovered following redo operation and anticoagulation. Hypercoagulable workup was negative, including factor $\mathrm{V}$ Leiden, prothrombin gene mutation, protein $\mathrm{C} / \mathrm{S}$, antithrombin III, homocysteine levels and antiphospholipid antibodies. He only had erythrocytosis with a hematocrit of $60 \% 1$ month prior to his presentation, which was present on admission with cardiogenic shock, but resolved 1 month postoperatively.

Connors et al [27] reported a 71-year-old male who was admitted with dyspnea due to BPVT and calcification of mitral valve prosthesis despite an international normalized ratio (INR) of 4; he recovered following redo MVR. His past medical history included parathyroidectomy, for which he was on daily calcium and vitamin D supplementation.

Rosa et al [28] reported a 74-year-old female with history of bioprosthetic MVR who developed extensive left atrial thrombi following replacement of warfarin with fondaparinux secondary to acutely abnormal liver function tests. After a negative workup which excluded a thrombophilic state, viral/ autoimmune liver disease, cancer or biliary duct pathology, warfarin was restarted and fondaparinux was stopped, with subsequent resolution of the left atrial thrombi.

Kounis et al [29], commenting on a TAVR valve leaflet thrombosis case followed by stroke 3 years following implantation, suggested that a potential unifying mechanism could be a delayed hypersensitivity reaction to one or more components of the implanted valve or drugs administered during the implantation procedure (Kounis syndrome).

In an in vitro study, Jahren et al [30] measured the pulsatile hemodynamics across bioprosthetic aortic valves inserted into compliant aortic root phantoms with symmetrical and patient-specific designs. They concluded that aortic root morphology affects blood flow dynamics in the aortic sinuses and may be a cause of BPVT, and recommended that patientspecific aortic roots be used when selecting and positioning prosthesis.

Yao et al [31] suggested that elevated gradients across a TAVR implanted within a degenerated surgically implanted bioprosthesis can be partially due to the non-distensible nature of bioprosthetic valves, resulting in an underexpansion of valve-in-valve (VIV) implant leading to accelerated degeneration and likely thrombosis of these valves. They reported that larger surgical valve size, supra-annular transcatheter heart valve type, and higher transcatheter valve implantation depth, were shown to reduce the incidence of elevated postVIV gradient.

Cremer and colleagues [32] discussed several potential mechanisms for early bioprosthetic valve failure based on operative and echocardiographic findings, and proposed a widened differential diagnosis including thrombosis (BPVT), pannus formation, early structural valve deterioration (SVD), endocarditis, patient prosthesis mismatch, and technical error.

\section{Diagnosis}

Several studies have demonstrated the utility of various diagnostic modalities in the diagnosis of BPVT. Criteria for the diagnosis have also been suggested for the various diagnostic modalities. Although an increase in the TVG across a prosthesis associated with symptoms is often the initial clue on TTE, further diagnostic modalities are usually required to better characterize the cause and extent of the TVG increase and guide therapy. Egbe and colleagues [13] suggested clinical and echocardiographic criteria including atrial fibrillation, subtherapeutic INR, TVG, cusp thickening and mobility (Table 2 , [13]). Applying these criteria to 138 patients yielded rates of $72 \%, 90 \%, 78 \%$, and $87 \%$ for sensitivity, specificity, and positive and negative predicted values for diagnosing BPVT, respectively.

Oliver et al [12] demonstrated the utility of TEE in the diagnosis of BPVT in the mitral position in 161 patients with clinical or TTE criteria of prosthetic malfunction. Homogenous, echodense masses were identified in 15 patients $(9 \%)$, 10 of whom had confirmed BPVT. 
Table 2. Egbe's criteria for BPVT Diagnosis [13]

$\geq 50 \%$ TVG increase from baseline within 5 years of surgery without high cardiac output

Increase cusp thickening ( $>2 \mathrm{~mm}$ ) especially on the downstream aspect of the valve

Abnormal cusp mobility

Paroxysmal atrial fibrillation

Subtherapeutic INR

BPVT: bioprosthetic valve thrombosis; TVG: transvalvular gradient; INR: international normalized ratio.

Muskula et al [33] reported an 84-year-old male with worsening dyspnea 3 years following bioprosthetic MVR, who was found to have severe mitral stenosis by TTE. TEE revealed marked thickening of the mitral bioprosthetic leaflets with limited mobility and a large left atrial thrombus. After 5 days of parenteral anticoagulation he underwent intraoperative TEE which revealed resolution of the mitral valve findings, obviating the need for redo MVR; he therefore underwent only resection of the left atrial thrombus and had an uneventful recovery.

Parro et al [34] analyzed 111 patients who underwent TEE for various reasons following bioprosthetic MVR, and reported on the utility of serial TEE studies in the diagnosis and follow-up following treatment of BPVT in four confirmed cases.

Waterbury et al [35] reported a 73-year-old patient with hemolysis 1 day post SAPIEN S3 TAVR; TEE revealed impingement on the valve stent by the native calcified annulus causing reduced opening and severe periprosthetic aortic regurgitation. Hemolysis resolved and the periprosthetic leak decreased to trivial following VIV implantation with another SAPIEN S3. They also reported a 64-year-old woman undergoing surgical MVR whose intraoperative TEE immediately post-surgery revealed reduced mobility in one leaflet despite therapeutic heparin administration. Both cases demonstrated the important role of TEE in identifying the mechanism of restricted leaflet motion and differentiating mechanical factors from BPVT.

TEE, however, may have its limitations in the evaluation of prosthetic valve thrombosis, especially mechanical prostheses, as demonstrated by Perazella et al [36].

Using cardiac CT, Dalen and colleagues [37] demonstrated the prevalence of HALT and reduced leaflet motion (RLM) in surgically implanted Perceval sutureless aortic valve bioprostheses, even in patients on anticoagulation. It has been hypothesized that HALT and RLM may be caused by subclinical leaflet thrombosis, leading to premature SVD and risk of thromboemboli [38].

Galaska et al [39] reported a 64-year-old asymptomatic patient with a high TVG across an aortic bioprosthesis implanted 6 months earlier, detected on routine TTE which did not adequately visualize the leaflets; a cardiac multidetector CT (MDCT) revealed thickening with limited mobility of the basal segments of the leaflets consistent with thrombosis, which resolved following low-molecular-weight heparin and later warfarin with significant decrease in the TVG.

Reviewing a meta-analysis of observational studies, Rashid et al [40] concluded that MDCT-defined aortic BPVT is associated with a significantly increased risk of stroke. They recommended prospective studies to examine whether this can be prevented or treated with pharmacological agents.

Tarzia et al [41] demonstrated the utility of gated 64-row MDCT in cardiac imaging of prosthetic valve thrombosis, helping to concurrently evaluate thrombus characteristics and exclude coronary artery disease.

\section{Management}

The level of anticoagulation recommended following bioprosthetic valve implantation remains controversial with regards to the adequacy of antiplatelet therapy alone versus the need to add anticoagulation with warfarin, especially early following surgery. Lee et al [42] retrospectively analyzed 479 patients who were 3 months after bioprosthetic AVR and greater than 60 years old in a single center over 10 years. They found the incidence of thromboembolism and bleeding were similar in warfarin and aspirin-treated groups, and recommended that if the patient does not have indications of warfarin, early antithrombotic therapy with aspirin only may be more feasible for elderly patients. Similarly, in a prospective, single-center, open-label, randomized controlled trial, Rafiq et al [43] reported data from 328 patients post bioprosthetic AVR (with or without coronary artery bypass grafting (CABG)) suggesting that, for the first 3 months following surgery, aspirin $150 \mathrm{mg}$ daily might be equally effective as warfarin (INR 2 - 3) in preventing thromboembolic events, but with less major bleedings. In an editorial, Stevens and Doty [44] commented that the long-term effects of subclinical events such as reduced leaflet motion and thrombus deposition on bioprosthetic valves remain unknown and require further study before abandoning the use of warfarin in such patients.

Van der Wall and colleagues [45] followed 402 patients 1 year after bioprosthetic AVR, and reported that acenocoumarol caused increased risk of bleeding and no reduction in thromboembolic events compared with antiplatelets, thereby recommending aspirin over vitamin $\mathrm{K}$ antagonists (VKAs) for postoperative thromboprophylaxis therapy.

Based on the results from the Society of Thoracic Surgeons Adult Cardiac Surgery National Database, Brennan et al [46] reported that overall risk of death and embolism was small in the first 3 months following bioprosthetic AVR. Despite the lower risk of such events in patients on aspirin and warfarin compared to aspirin alone, this strategy was associated with an increased risk of bleeding.

Mydin et al [47] reviewed multiple studies on the optimal anticoagulation of bioprosthetic AVR and concluded that guidelines remain contradictory due to the limitations of avail- 
able studies with conflicting results. Until more definitive data are available, they suggested, critical assessment of the available literature will remain an important driver for decisionmaking and good clinical practice in thromboprophylaxis following bioprosthetic AVR.

Del Trigo et al [48] reported the results of a multicenter study of TVG by echocardiography 12 months following TAVR in 2,466 patients, 707 of whom received anticoagulation. They concluded that the lack of anticoagulation post TAVR was associated with significant increase in TVG and greater risk of valve hemodynamic deterioration, which was subclinical in most cases and did not cause major adverse clinical events.

Sanaani et al [49] reviewed the options for anticoagulation in patients with prosthetic valves and emphasized the risk of early bioprosthetic valve thrombosis, especially in the mitral position, and the need for warfarin within 3 months of insertion; they remarked that no trials to date have reported the efficacy and safety of novel oral anticoagulants within 3 months of bioprosthetic valve implantation.

Thourani and colleagues [50] retrospectively analyzed 572 patients who underwent bioprosthetic MVR over a 5-year period with regards to treatment with either $4-6$ weeks of postoperative warfarin or no warfarin. They reported that although the use of postoperative warfarin did not reduce the incidence of stroke at early follow-up, it was associated with a trend for improved long-term outcomes.

The 2017 AHA/ACC Focused Update of the 2014 AHA/ ACC Guideline for the Management of Patients With Valvular Heart Disease [51] recommended that for both aortic and mitral bioprosthetic valves, in addition to aspirin, anticoagulation with warfarin targeting an INR of 2.5 should be considered (IIa recommendation) in patients with a low risk of bleeding for 3 to 6 months following surgery. The guidelines further recommended that after TAVR it is reasonable (IIb recommendation) to use anticoagulation with warfarin targeting an INR of 2.5 for at least 3 months, or clopidogrel $75 \mathrm{mg}$ daily for the first 6 months in addition to life-long aspirin $75 \mathrm{mg}$ to $100 \mathrm{mg}$ daily.

The management of prosthetic valve thrombosis varies according to the type of prosthesis involved, the size of the thrombus and the severity of the hemodynamic abnormalities. Monteiga and colleagues [52] reported an overall $82 \%$ success rate with the use of thrombolytics in prosthetic valve thrombosis in various positions. Lengyel et al [53] analyzed several studies on prosthetic valve thrombosis and recommended that thrombolysis, followed by heparin, warfarin and aspirin, were advised for high risk surgical candidates with left-sided prosthetic valve thrombosis.

Comparing BPVT patients treated initially with warfarin versus surgery/thrombolysis, Pislaru et al [54] reported that warfarin resulted in hemodynamic and clinical improvement with minimal risk. They recommended warfarin as first-line therapy in hemodynamically stable patients with BPVT.

Butnaru et al [55] reported an incidence of $6 \%$ mitral BPVT out of 149 MVR operations over 10-year period, twothirds of whom showed resolution of symptoms and TVG with anticoagulation, while one-third required surgery. They recommended that an initial trial with anticoagulation is warranted in this condition, and that surgery should be reserved for those who are hemodynamically unstable. They noted that non-resection of the native valve at the initial operation may play a role in the presence of BPVT.

Alshehri et al [56] reported a 26-year-old female with a porcine mitral bioprosthesis who presented with acute pulmonary edema secondary to obstructive valve thrombosis. A favorable outcome was observed after conservative anticoagulant treatment.

Some cases of BPVT involve a large thrombus burden not responsive to anticoagulants or thrombolysis, requiring valve reoperation or thrombectomy. Tarzia et al [57] reported a 73-year-old male who presented with dyspnea and elevated TVG 1 year following aortic valve and ascending aorta replacement. He required re-operation, which revealed an intact valve with massive thrombus treated successfully with thrombectomy. Fan et al [58] reported a similar case in a 61-year-old male who presented with cardiogenic shock 11 months following a bioprosthetic porcine AVR, requiring surgery. He was found to have thrombosis of the three cusps on the aortic side, limiting the opening of the valve, and necessitating thrombectomy.

Jung et al [59] reported a 77-year-old female who presented with progressive dyspnea 2 years following bioprosthetic MVR due to BPVT unresponsive to thrombolytic therapy and diuresis. She underwent successful urgent redo MVR with a bioprosthesis.

The duration of anticoagulation following an episode of BPVT is unclear; however, Yong et al [60] described a case of recurrence of BPVT 6 months following cessation of anticoagulation in a successfully treated aortic BPVT and recommended life-long anticoagulation in this patient group. The increasing use of TAVR as an alternative to surgical aortic valve replacement in various risk groups has introduced new challenges with regards to TAVR BPVT prevalence [61], evaluation and imaging [62], etiologies [63], cerebrovascular [64] and cardiac [65] embolic consequences, adequate treatment with warfarin versus NOACs $[66,67]$, intermediate and long-term anticoagulation [68] and follow-up [69]. The increasing use of valve-in-valve procedures is expected to bring on further uncharted challenges [70].

\section{Conclusions}

BPVT is a major cause of either acute or indolent bioprosthetic valve degeneration, and often has an elusive presentation causing delayed recognition and treatment. The literature has extensively addressed the risks and benefits of anticoagulation following bioprosthetic valve replacement to prevent BPVT, without conclusive results or evidence-based recommendations. It seems that the exponential research and clinical use of catheter-based and to a lesser extent, surgical-based, valve therapies have not been paralleled with commensurate research in the required adjunctive antithrombotic and anticoagulant therapies to assure safe delivery and long-term outcomes of such therapies, especially with regards to BPVT.

\section{Financial Support}

None. 


\section{Conflict of Interest}

None.

\section{References}

1. Puri R, Auffret V, Rodes-Cabau J. Bioprosthetic Valve Thrombosis. J Am Coll Cardiol. 2017;69(17):2193-2211.

2. Bax JJ, Stone GW. Bioprosthetic surgical and transcatheter heart valve thrombosis. Lancet. 2017;389(10087):23522354.

3. Barlow CW. Bioprosthetic valve thrombosis: Are we not seeing the wood for the trees? J Thorac Cardiovasc Surg. 2016;152(4):978-980.

4. Iung B, Vahanian A. Epidemiology of valvular heart disease in the adult. Nat Rev Cardiol. 2011;8(3):162-172.

5. Dangas GD, Weitz JI, Giustino G, Makkar R, Mehran R. Prosthetic Heart Valve Thrombosis. J Am Coll Cardiol. 2016;68(24):2670-2689.

6. Egbe AC, Connolly HM, Schaff HV. Bioprosthetic valve thrombosis: What we know and what we need to know. J Thorac Cardiovasc Surg. 2016;152(4):975-978.

7. Ad N. Bioprosthetic valve thrombosis following surgical aortic valve replacement: Did we all miss it? J Thorac Cardiovasc Surg. 2016;152(4):981-982.

8. Brown ML, Park SJ, Sundt TM, Schaff HV. Early thrombosis risk in patients with biologic valves in the aortic position. J Thorac Cardiovasc Surg. 2012;144(1):108-111.

9. Makkar RR, Fontana G, Jilaihawi H, Chakravarty T, Kofoed KF, De Backer O, Asch FM, et al. Possible subclinical leaflet thrombosis in bioprosthetic aortic valves. N Engl J Med. 2015;373(21):2015-2024.

10. Chakravarty T, Sondergaard L, Friedman J, De Backer O, Berman D, Kofoed KF, Jilaihawi H, et al. Subclinical leaflet thrombosis in surgical and transcatheter bioprosthetic aortic valves: an observational study. Lancet. 2017;389(10087):2383-2392.

11. Cordoba-Soriano JG, Puri R, Amat-Santos I, Ribeiro HB, Abdul-Jawad Altisent O, del Trigo M, Paradis JM, et al. Valve thrombosis following transcatheter aortic valve implantation: a systematic review. Rev Esp Cardiol (Engl Ed). 2015;68(3):198-204.

12. Oliver JM, Gallego P, Gonzalez A, Dominguez FJ, Gamallo C, Mesa JM. Bioprosthetic mitral valve thrombosis: clinical profile, transesophageal echocardiographic features, and follow-up after anticoagulant therapy. J Am Soc Echocardiogr. 1996;9(5):691-699.

13. Egbe AC, Pislaru SV, Pellikka PA, Poterucha JT, Schaff HV, Maleszewski JJ, Connolly HM. Bioprosthetic Valve Thrombosis Versus Structural Failure: Clinical and Echocardiographic Predictors. J Am Coll Cardiol. 2015;66(21):2285-2294.

14. Yanagawa B, Mazine A, Bhatt DL, Clavel MA, Cote N, Cheema AN, Pibarot P, et al. Subclinical bioprosthetic aortic valve thrombosis: clinical and translational implications. Curr Opin Cardiol. 2017;32(2):137-146.

15. Sondergaard L, De Backer O, Kofoed KF, Jilaihawi H,
Fuchs A, Chakravarty T, Kashif M, et al. Natural history of subclinical leaflet thrombosis affecting motion in bioprosthetic aortic valves. Eur Heart J. 2017;38(28):22012207.

16. Doris MK, Dweck MR. Is bioprosthetic leaflet thrombosis a trigger to valve degeneration? Heart. 2018;104(10):792793.

17. Mack M, Holmes D. Bioprosthetic valve thrombosis: The harder one looks, the more one finds. J Thorac Cardiovasc Surg. 2016;152(4):952-953.

18. Imada T, Shibata SC, Okitsu K, Fujino Y. Unexpected bioprosthetic mitral valve thrombus during left ventricular assist device implantation. JA Clin Rep. 2017;3(1):15.

19. Madathil R, Togashi K, VonHomeyer P, Hermsen JL. Acute bioprosthetic mitral valve thrombosis. Intensive Care Med. 2016;42(6):1073-1074.

20. Kagiyama N, Okura H, Nezuo S, Kawamoto T, Murakami T, Hashimoto Y, Tanemoto K, et al. Two cases of acute bioprosthetic mitral valve thrombosis immediately after mitral valve replacement. Circulation. 2014;129(6):e328330 .

21. Kothari SS, Deepti S, Rai N. Reversible bioprosthetic valve thrombosis from eosinophilia. BMJ Case Rep. 2018;2018.

22. Yesin M, Kalcik M, Gunduz S, Astarcioglu MA, Gursoy MO, Karakoyun S, Ozkan M. Bioprostethic mitral valve thrombosis due to oral contraceptive drug use and management with ultra-slow thrombolytic therapy. Blood Coagul Fibrinolysis. 2016;27(2):220-222.

23. Poels TT, Veenstra L, van Ommen V, Kietselaer BL, Das M, Maessen JG, Kats S. Severely thrombosed transcatheter aortic valve 9 months after implantation. Ann Thorac Surg. 2015;100(4):1441-1444.

24. Chamsi-Pasha MA, Alyousef T, Sayyed S. Bioprosthetic mitral valve thrombosis complicating antiphospholipid antibody syndrome, successfully treated with thrombolysis. Echocardiography. 2014;31(9):E278-281.

25. Sidhu M, Goel P, Singh HP, Chopra AK, Arora R, Sidhu $\mathrm{S}$. Acute bioprosthetic mitral valve stenosis in a patient with HITS. J Card Surg. 2011;26(4):369-371.

26. Tsiouris A, Nemeh H, Borgi J. Early acute thrombosis of bioprosthetic mitral valve presenting with cardiogenic shock. Gen Thorac Cardiovasc Surg. 2013;61(3):152-154.

27. Connors CW, Christie AA, Weldner PW. Early degeneration of a bioprosthetic mitral valve complicated by a large left atrial thrombus. Semin Cardiothorac Vasc Anesth. 2011;15(3):112-114.

28. Rosa GM, Parodi A, Dorighi U, Carbone F, Mach F, Quercioli A, Montecucco F, et al. Left atrial thrombosis in an anticoagulated patient after bioprosthetic valve replacement: Report of a case. World J Clin Cases. 2014;2(1):20-23.

29. Kounis NG, Grapsas N, Soufras GD, Lianas D, Patsouras $\mathrm{N}$, Hahalis G. Clinical and subclinical leaflet thrombosis in bioprosthetic aortic valves: A manifestation of Kounis syndrome? Int J Cardiol. 2016;214:86-87.

30. Jahren SE, Heinisch PP, Hasler D, Winkler BM, Stortecky S, Pilgrim T, Londono MC, et al. Can bioprosthetic valve thrombosis be promoted by aortic root morphol- 
ogy? An in vitro study. Interact Cardiovasc Thorac Surg. 2018;27(1):108-115.

31. Yao RJ, Simonato M, Dvir D. Optimising the Haemodynamics of Aortic Valve-in-valve Procedures. Interv Cardiol. 2017;12(1):40-43.

32. Cremer PC, Rodriguez LL, Griffin BP, Tan CD, Rodriguez ER, Johnston DR, Pettersson GB, et al. Early bioprosthetic valve failure: mechanistic insights via correlation between echocardiographic and operative findings. J Am Soc Echocardiogr. 2015;28(10):1131-1148.

33. Muskula PR, Ramirez R, Borkon AM, Main ML. Late thrombosis of a mitral bioprosthetic valve with associated massive left atrial thrombus. Echo Res Pract. 2017;4(1):I1-I3.

34. Parro A, Jr., Carramona ML, Amaral CA, Jacob JL, Nicolau JC. Bioprosthetic mitral valve thrombosis. Importance of transesophageal echocardiography in the diagnosis and follow-up after treatment. Arq Bras Cardiol. 2004;82(4):346-359.

35. Waterbury TM, Raphael CE, Padang R, Eleid MF, Holmes DR, Jr., Rihal CS, Pislaru SV. Not all immobile bioprosthetic valve cusps are thrombosed. JACC Cardiovasc Interv. 2017;10(12):e117-e118.

36. Perazella MA, Frederick W, Buller GK, Widman S. Mechanical mitral valve prosthesis dysfunction from thrombus: transesophageal echocardiography has limitations. Conn Med. 1996;60(2):75-77.

37. Dalen M, Sartipy U, Cederlund K, Franco-Cereceda A, Svensson A, Themudo R, Svenarud P, et al. Hypo-Attenuated Leaflet Thickening and Reduced Leaflet Motion in Sutureless Bioprosthetic Aortic Valves. J Am Heart Assoc. 2017;6(8):e005251.

38. Yanagawa B, Verma S, Mazer CD. Subclinical Valve Thrombosis in Sutureless Bioprosthetic Valves. J Am Heart Assoc. 2017;6(8):e006862.

39. Galaska R, Kulawiak-Galaska D, Fijalkowski M, Szurowska E, Gruchala M. Multidetector computed tomography to detect reversible subclinical aortic bioprosthetic valve thrombosis with high systolic gradients. Cardiol J. 2016;23(4):411-412.

40. Rashid HN, Gooley RP, Nerlekar N, Ihdayhid AR, McCormick LM, Nasis A, Cameron JD, et al. Bioprosthetic aortic valve leaflet thrombosis detected by multidetector computed tomography is associated with adverse cerebrovascular events: a meta-analysis of observational studies. EuroIntervention. 2018;13(15):e1748-e1755.

41. Tarzia V, Bortolussi G, Rubino M, Gallo M, Bottio T, Gerosa G. Evaluation of prosthetic valve thrombosis by 64-row multi-detector computed tomography. J Heart Valve Dis. 2015;24(2):210-213.

42. Lee SI, Lee KS, Kim JB, Choo SJ, Chung CH, Lee JW, Jung SH. Early Antithrombotic Therapy after Bioprosthetic Aortic Valve Replacement in Elderly Patients: A Single-Center Experience. Ann Thorac Cardiovasc Surg. 2017;23(3):128-134.

43. Rafiq S, Steinbruchel DA, Lilleor NB, Moller CH, Lund JT, Thiis JJ, Kober L, et al. Antithrombotic therapy after bioprosthetic aortic valve implantation: Warfarin versus aspirin, a randomized controlled trial. Thromb Res.
2017; 150:104-110.

44. Stevens SM, Doty JR. New evidence on old drugs; warfarin versus aspirin after bioprosthetic aortic valve placement. Thromb Res. 2017;150:102-103.

45. van der Wall SJ, Umans VA, Schotten J, Keijzers M, Wolterbeek R, Jansen EK, Huisman MV, et al. Antithrombotic strategy after bioprosthetic aortic valve replacement in patients in sinus rhythm: evaluation of guideline implementation. Eur J Cardiothorac Surg. 2016;49(4):11571163.

46. Brennan JM, Edwards FH, Zhao Y, O'Brien S, Booth ME, Dokholyan RS, Douglas PS, et al. Early anticoagulation of bioprosthetic aortic valves in older patients: results from the Society of Thoracic Surgeons Adult Cardiac Surgery National Database. J Am Coll Cardiol. 2012;60(11):971977.

47. Mydin MI, Dimitrakakis G, Younis J, Nowell J, Athanasiou T, Kourliouros A. Optimal thromboprophylaxis following bioprosthetic aortic valve replacement: still a matter of debate? Interact Cardiovasc Thorac Surg. 2012;15(1):109-114.

48. Del Trigo M, Munoz-Garcia AJ, Latib A, Auffret V, Wijeysundera HC, Nombela-Franco L, Gutierrez E, et al. Impact of anticoagulation therapy on valve haemodynamic deterioration following transcatheter aortic valve replacement. Heart. 2018;104(10):814-820.

49. Sanaani A, Yandrapalli S, Harburger JM. Antithrombotic management of patients with prosthetic heart valves. Cardiol Rev. 2018;26(4):177-186.

50. Thourani VH, Gunter RL, Hurst S, Kilgo P, Padala M, Puskas JD, Lattouf OM, et al. Postoperative warfarin following mitral valve repair or bioprosthetic valve replacement. J Heart Valve Dis. 2013;22(5):716-723.

51. Nishimura RA, Otto CM, Bonow RO, Carabello BA, Erwin JP, 3rd, Fleisher LA, Jneid H, et al. 2017 AHA/ACC Focused Update of the 2014 AHA/ACC Guideline for the management of patients with valvular heart disease: a report of the American College of Cardiology/American Heart Association Task Force on clinical practice guidelines. J Am Coll Cardiol. 2017;70(2):252-289.

52. Manteiga R, Carlos Souto J, Altes A, Mateo J, Aris A, Dominguez JM, Borras X, et al. Short-course thrombolysis as the first line of therapy for cardiac valve thrombosis. J Thorac Cardiovasc Surg. 1998;115(4):780-784.

53. Lengyel M, Fuster V, Keltai M, Roudaut R, Schulte HD, Seward JB, Chesebro JH, et al. Guidelines for management of left-sided prosthetic valve thrombosis: a role for thrombolytic therapy. Consensus Conference on Prosthetic Valve Thrombosis. J Am Coll Cardiol. 1997;30(6):1521-1526.

54. Pislaru SV, Hussain I, Pellikka PA, Maleszewski JJ, Hanna RD, Schaff HV, Connolly HM. Misconceptions, diagnostic challenges and treatment opportunities in bioprosthetic valve thrombosis: lessons from a case series. Eur J Cardiothorac Surg. 2015;47(4):725-732.

55. Butnaru A, Shaheen J, Tzivoni D, Tauber R, Bitran D, Silberman S. Diagnosis and treatment of early bioprosthetic malfunction in the mitral valve position due to thrombus formation. Am J Cardiol. 2013;112(9):1439-1444. 
56. Alshehri HZ, Ismail M, Ibrahim MF. Obstructive bioprosthetic mitral valve thrombus: management options? Asian Cardiovasc Thorac Ann. 2014;22(8):975-978.

57. Tarzia V, Dal Lin C, Bottio T, Gerosa G. Thrombectomy for massive bioprosthetic valve thrombosis. Eur J Cardiothorac Surg. 2011;40(6):1540.

58. Fan J, Teng P, Zou Y, Ma L. A rare case report of early bioprosthetic valve thrombosis presenting with acute heart failure salvaged by thrombectomy. J Cardiothorac Surg. 2017;12(1):19.

59. Jung HJ, Song BG, Heo SH, Kim KY, Kim YI. Catastrophic late mitral bioprosthetic thrombosis. Asian Cardiovasc Thorac Ann. 2012;20(1):61-63.

60. Yong MS, Grant R, Saxena P, Yadav S. Recurrent bioprosthetic valve thrombosis - should long-term anticoagulation be considered? Heart Lung Circ. 2018;27(6):e70e72.

61. Rashid HN, Nasis A, Gooley RP, Cameron JD, Brown AJ. The prevalence of computed tomography-defined leaflet thrombosis in intra- versus supra-annular transcatheter aortic valve prostheses. Catheter Cardiovasc Interv. 2018.

62. Zhao ZG, Wang MY, Jilaihawi H. Imaging Evaluation for the Detection of Leaflet Thrombosis After Transcatheter Aortic Valve Replacement. Interv Cardiol Clin. 2018;7(3):293-299.

63. Deutsch MA, Scotten LN, Siegel R, Lange R, Bleiziffer S. Leaflet thrombosis and clinical events after TAVR: are paravalvular leaks a crucial trigger? EuroIntervention. 2018;14(6):716-717.
64. Makki N, Shreenivas S, Kereiakes D, Lilly S. A metaanalysis of reduced leaflet motion for surgical and transcatheter aortic valves: Relationship to cerebrovascular events and valve degeneration. Cardiovasc Revasc Med. 2018.

65. Li YJ, Liao YB, Wei X, Feng Y, Chen M. Acute Myocardial Infarction as the Initial Manifestation of Delayed Bioprosthesis Thrombosis After Transcatheter Aortic Valve Replacement. Heart Lung Circ. 2018;27(4):e46-e50.

66. Khan S, Achike O, Nekkanti R, Carabello B. Successful Apixaban Treatment of TAVR Thrombosis after Warfarin Failure. Cardiology. 2018;140(4):199-203.

67. Mathiassen ON, Grove EL, Hansson NC, Norgaard BL. Late Obstructive Transcatheter Heart Valve Thrombosis Resolved by Rivaroxaban. Am J Case Rep. 2017;18:573575.

68. Sorrentino S, Giustino G, Moalem K, Indolfi C, Mehran R, Dangas GD. Antithrombotic Treatment after Transcatheter Heart Valves Implant. Semin Thromb Hemost. 2018;44(1):38-45.

69. Ruile P, Minners J, Breitbart P, Schoechlin S, Gick M, Pache G, Neumann FJ, et al. Medium-Term FollowUp of Early Leaflet Thrombosis After Transcatheter Aortic Valve Replacement. JACC Cardiovasc Interv. 2018;11(12):1164-1171.

70. Wernly B, Zappe AK, Unbehaun A, Sinning JM, Jung C, Kim WK, Fichtlscherer S, et al. Transcatheter valve-invalve implantation (VinV-TAVR) for failed surgical aortic bioprosthetic valves. Clin Res Cardiol. 2018. 